\title{
Mechanisms of drug action
}

\section{The molecular basis of drug toxicity}

\author{
W.D.M.PATON \\ From the University Department of Pharmacology, Oxford
}

This paper must necessarily be highly selective in an enormous field, and will represent my own attempts to come to grips with a particular group of pharmacological actions rather than to cover a wide field. Briefly, I would like to review the basis for the distinction of specific from non-specific pharmacological action, and, taking anaesthetics as the prototypes of non-specific action, use them as guides to the properties of fat-soluble (lipophilic) substances generally. Finally, I would like to consider ways in which unitary theories of anaesthesia fail or need adjustment and the types of mechanisms which may be involved. From this review a possible general pattern of lipophilic toxicity emerges.

\section{The Distinction between Specific and Nonspecific Actions}

The classic examples of specific action are found in drugs used in the autonomicfield. The tablelists some of the characteristics of these compounds which have generated the idea of the 'drug receptor', that is, an array of spatially organized sites interacting with details of the structure of the drug in the same way as a substrate does with its enzyme. These are by no means the only examples; the toxicity of fluoracetate, or of nitrosamines, the release of histamine by amines, the action of antibiotics, the reactions leading to sensitization to chemicals, and many others, all lead to pictures full of molecular detail.

General anaesthetics can be contrasted with all these. Thus, the chemical structures of active compounds lack specificity, ranging from the inert xenon through ether, chloroform, nitrous oxide, barbiturates, chloralose, to the steroid anaesthetics. Further, although there is a characteristic (though variable) progression of action with increasing dose from depression of higher centres downwards through the central nervous system and the body, yet almost every system or tissue is affected by concentrations of anaesthetic spanning only three- to five-fold differences. This differs considerably from the specificity of 1000 -fold or more with, for example, some autonomic drugs, or penicillin. It is necessary to think differently and rather generally about anaesthetics, to accommodate both the extreme diversity of chemical structures and the unspecificity of effects. In fact, there are also other areas in pharmacology where a number of different compounds seem to produce the same general pattern of action: very many amines, for instance, will produce local anaesthesia and will act as anti-histamines, anti-adrenalines, or antiacetylcholines in a modest way. Such observations underlie the remark that 'any drug will do anything' which is a rather lightweight saying comparable to 'any man is capable of any action'; doubtless true enough, yet we are in practice selective, and not many of us practise burglary! The important point is the inductive hint that there may be patterns of action which are not tightly coupled to detailed chemical structure.

\section{Mechanisms of Anaesthesia}

The best understood of these patterns is that of the general anaesthetics, and I think it is worth discussing this briefly. It is over 70 years since Overton and Meyer suggested that these were linked, not by chemical structure, but by the physical property of fat solubility; this produced the generalization that any

Existence of pairs of specific, and structurally related, agonists and antagonists (eg, propranolol/isoprenaline)

High potency (eg, acetylcholine/hyoscine) and high affinity when it is possible to determine it

Potency strongly dependent on small details of chemical structure (eg, noradrenaline)

Potency dependent on optical activity (eg, 1-adrenaline vs d-adrenaline)

Activity strongly restricted to particular cells or parts of cells (eg, acetylcholine/tubocurarine)

Appropriate kinetic relationships between concentrations of agonist and antagonist producing a given effect (eg, mepyramine/histamıne)

Evidence for specific binding to receptor sites (eg, atropine, bungarotoxin)

Table Characteristics of specific drug action illustrated by agonists and antagonists in the autonomic, neuromuscular and histamine fields 
substance would cause anaesthesia if, when given in a suitable concentration, it dissolved in fat to a level of about $0.05 \mathrm{M}$. A highly fat-soluble substance needs only a low inhaled concentration to yield the required level in fat and less soluble compounds are correspondingly less potent. This generalization for a long time remained just one of many. Indeed it was explicitly questioned by the clathrate theory of anaesthesia; this suggested that the intermolecular forces leading to solubility in fat would also lead to a characteristic structuring of water round the anaesthetic molecule and cause an interference with water-based biological processes. In this theory, there was a major difference of approach, the important events of anaesthesia taking place in a watery, not a fatty, environment. However, when fuller and better correlations were made of anaesthetic potency with water structuring on the one hand, and fat solubility on the other, particularly using modern (and conveniently anomalous) fluorine-containing compounds, it became clear that fat solubility was much the best candidate (fig 1). Furthermore, two other lines developed: it was possible to show that anaesthetics, dissolved in artificial or natural membranes, produced a 'fluidification' of the lipid of the membrane (Burgen and Metcalfe, 1970), together with evidence of a slight but definite expansion. Moreover, an old observation that very high pressure (around 100 atmospheres or $3000 \mathrm{ft}$ of sea water) would reverse anaesthesia was re-examined and confirmed (Lever et al, 1971) (fig 2), with evidence that the compression so produced just about balanced quantitatively the expansion by the anaesthetic (Miller et al, 1973), and restored the normal mobility of

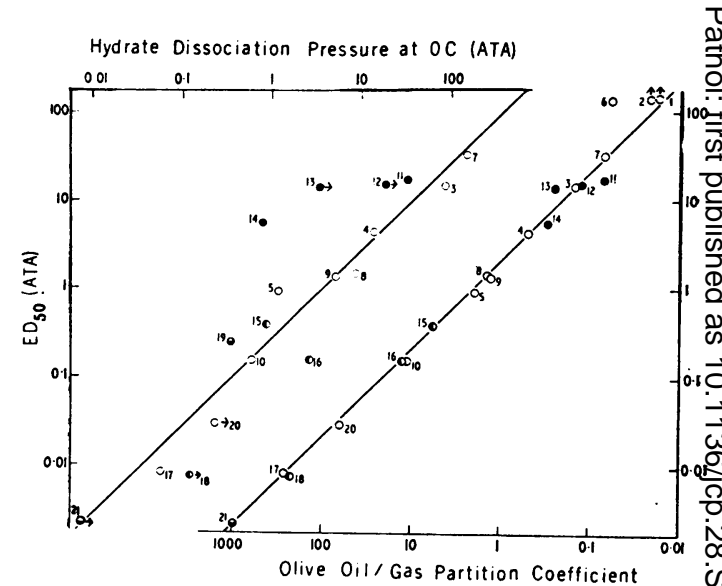

Fig 1 The correlation of anaesthetic pressure (in atmospheres absolute, ATA) with hydrate dissociation pressure $(A T A)$ at $0^{\circ} \mathrm{C}$ (left-hand graph) compared with the correlation of anaesthetic potency with the olive-oil partition coefficient at $37^{\circ} \mathrm{C}$. Solid black dots are fully fluorinated molecules; partially black dots are partially fluorinated molecules (from Miller, Paton, Smith, and Smith, 1972, Anesthiology, 36, 339-351).

the membrane (Trudell, Hubbell, and Cohen, 1973)

It would be inappropriate to discuss further deepe opments, but the success of fat solubility theories enabled workers to focus more confidently on hydif phobic regions as the sites of action for such mofles cules. My own guess, at least so far as anaesthesia $\frac{D}{6}$ concerned, is that the site of action of such suls stances is primarily the limiting membranes of nerve cells and these should be viewed in the light of modern
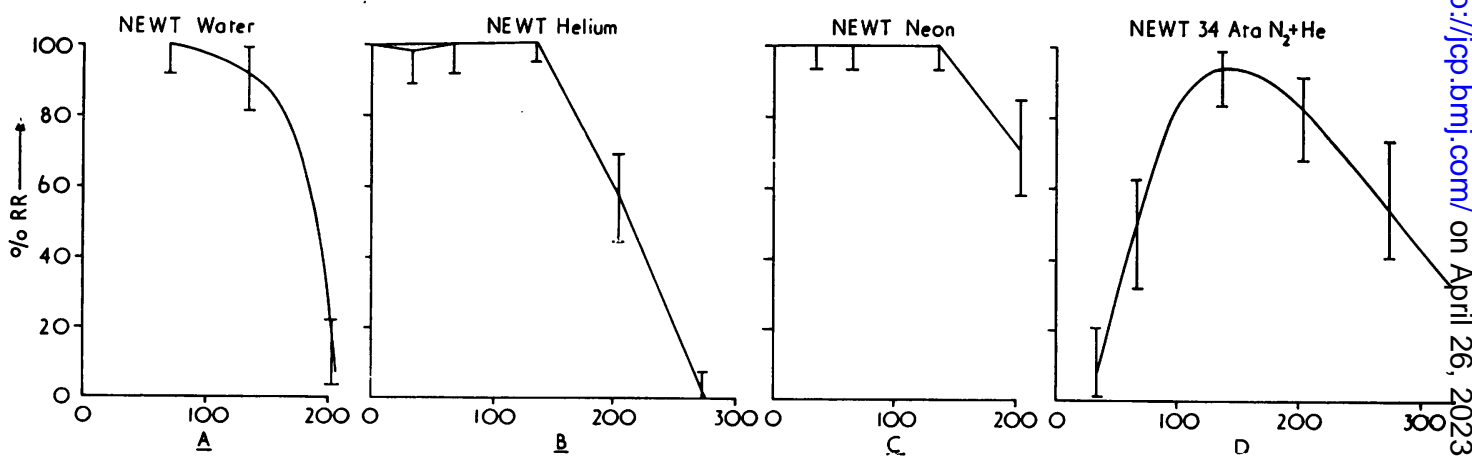

Fig 2 Rolling response of newts (ordinate: \% successful righting) under various conditions (abscissa: pressure of water or gas in atmospheres): $\mathrm{A}$, exposed to hydrostatic pressure only; $\mathrm{B}$, exposed to helium; $\mathrm{C}$, exposed to neon; $\mathrm{D}$, exposed to 34 ATA nitrogen to produce anaesthesia and then helium.

Comparison of $\mathrm{A}, \mathrm{B}$ and $\mathrm{C}$ show that the effects of helium and neon are those of pressure per se; in $\mathrm{D}$ the application of high pressure by helium antagonizes the anaesthesia produced by nitrogen (from Lever, Miller, Paton, and Smith, 1971, Nature (Lond.), 231, 368-371). 


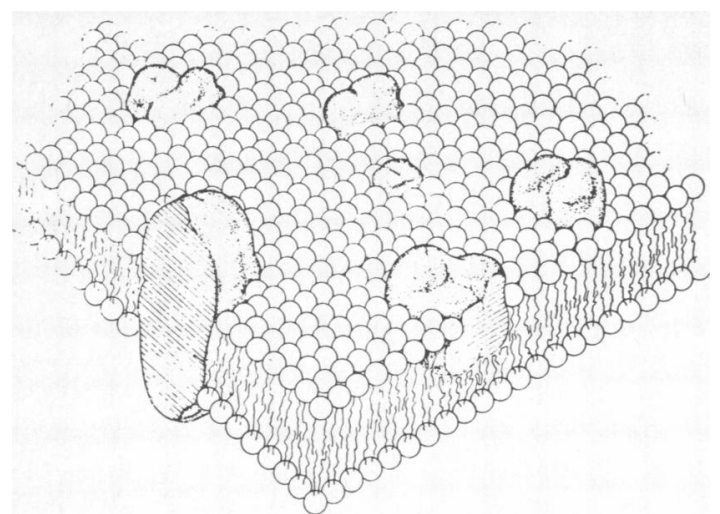

Fig 3 The lipid-globular protein mosaic model with a lipid matrix (the fluid mosaic model); schematic threedimensional and cross-sectional views. The solid bodies with stippled surfaces represent the globular integral proteins, which at long range are randomly distributed in the plane of the membrane. At short range, some may form specific aggregates, as shown (from Singer and Nicolson, 1972, Science, 175, 720-731. Copyright 1972 by the American Association for the Advancement of Science).

ideas of the structure of the cell membranes (fig 3). Intracellular membranes or the hydrophobic interior of protein macromolecules may well also be important. But although investigators now join in concentrating on hydrophobic regions, there is great uncertainty and a diversity of views on the more detailed molecular theory. The 'critical volume hypothesis' that our work at Oxford has supported and which says that anaesthesia occurs when insertion of a lipophilic molecule into the hydrophobic region critical for anaesthesia leads to a critical increase in its volume hardly provides a mechanism. The increased mobility of lipid chains, which would of course involve membrane expansion, could be envisaged as changing membrane permeability; but we also know that with lipophiles of higher molecular weight, order rather than disorder of the membrane occurs, although volume increases would still be expected. Consequently a considerable range of possibilities is being considered (see the symposium edited by Halsey et al, 1974): perturbation of the structure of the membrane (such that any deviation in either direction from normal ordering impairs function); perturbation at the site of insertion of macromolecules into the membrane, or propagation of disturdance from the lipid layers to the macromolecule; action within the macromolecule itself; interaction with intracellular membranes, eg, neurofilaments and tubules; interactions with mitochondrial membranes leading to metabolic changes, or to failure of $\mathrm{Ca}^{++}$ sequestration.

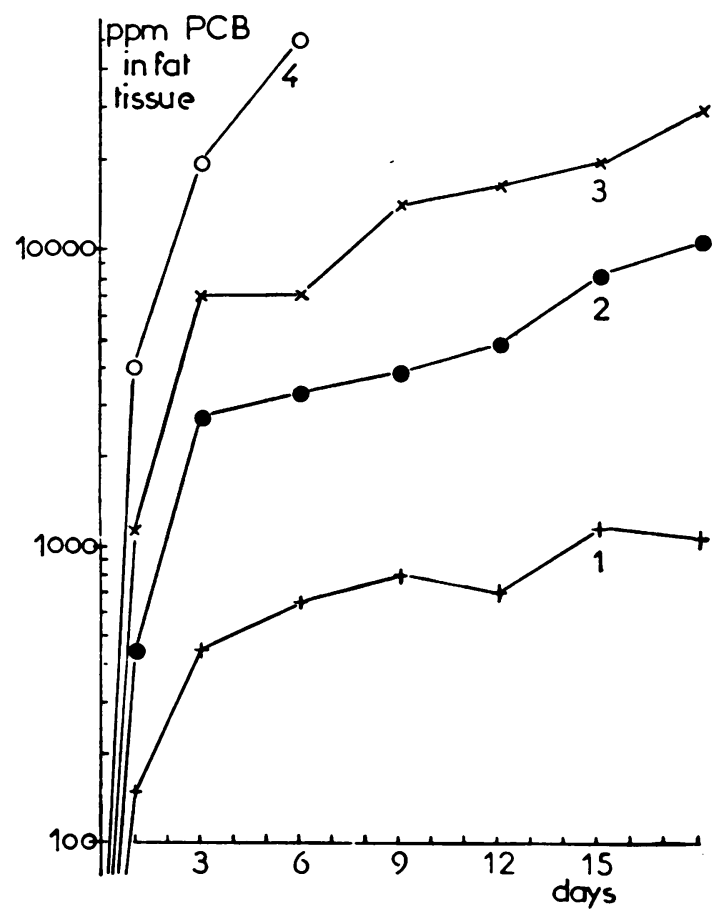

Fig 4 Absorption of $P C B$ by goldfish from different concentrations of $P C B$ in water. Abscissa: Time in days. Ordinate: Concentration of PCB in fat tissue (ppm in log scale). Water concentration of $P C B$ :

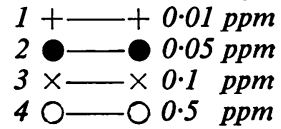

(from Hattula and Karlog, 1973, Acta pharmacol. toxicol., 32, 237-245).

Now all these are theories but there is evidence for each of them, and I think that they deserve attention, not just in connexion with clinical anaesthesia but in connexion with the action of any fat-soluble substance. Further, although anaesthesia is normally a short-term affair, chronic exposure may occur with other lipophiles and continued actions, of the types mentioned, even at a low-grade level, may present new features. Such possibilities must be taken seriously, because there is now something of a convergence of interest in lipophilic toxicity. For example, the toxicity of DDT, chlorinated biphenyls, hexachlorophane, industrial solvents, vinyl chloride, cannabis, the constituents of fuel oil in alcoholic drinks and the chronic effects of anaesthetics, are obvious cases. It is quite pretty to see, for instance, how polychlorinated biphenyls(PCB) are partitioned, as one would expect from simple physical principles, 
into fat in fish (fig 4). I suspect, too, that a large range of other substances, such as amines, should also be included as they can exist in an uncharged lipid-soluble form. Although their ionizable groups must influence their actions and their distribution enormously, nevertheless a substantial hydrophobic component in the molecule could well bring about an anaesthetic-type action, at least at higher doses; one recalls that chlorpromazine can itself be used to produce anaesthesia and that poisoning with tricyclic antidepressants is a puzzling and refractory condition.

\section{Possible Patterns of Lipophilic Toxicity}

In attempting to map out the possible patterns of toxicity of a lipophile, one has to move from an inferred mechanism of action to possible patterns of effects. This is the reverse of the normal procedure and is made necessary by the lack of detailed toxicological study. It is probable that chronic toxicity will be more important than acute, simply because the elimination time is likely to be prolonged for a substance which segregates in fatty phases of the body, away from the water-based excretory system. There is one major qualification and that is in the liver. The microsomal system of this organ can be viewed as a mechanism for rendering fat-soluble chemicals more water-soluble (for instance by hydroxylation) and hence accelerating elimination (Parke, 1968). Consequently it is necessary to know the final balance between segregation and metabolism. If retention in fact occurs, then continued dosage would give much higher tissue levels than one would guess from single-dose studies and the latter would not be a reliable guide to toxic effects.

There is little guidance as to the likely pattern, either because many chemicals have never been studied chronically or (when they have) the question has usually been to determine a non-toxic level rather than to study the pattern of phenomena at a toxic level. However, there are a number of recurrent themes, if one draws on animal work with chronic exposure to anaesthetics, on some classical toxicology and on recent work with cannabis which is a highly lipophilic substance and is often administered chronically (see Mechoulam, 1973).

I shall start by being too 'unitary' and go on to a number of qualifications.

First, it has been known for some time that continued exposure to nitrous oxide can produce aplastic anaemia in animals (see Fink, 1968). This is one example of the ability of lipophiles to interfere with cell division. This may show itself directly, or as immunodepression, or as induction of reduction deformity in the fetus, or as interference with sperma togenesis.

Second, there is a range of biochemical effects. including the uncoupling of oxidative phosphorylap tion, interference with NADH reoxidation, and inter ference with protein and nucleic acid synthesis.

Third, there is an interesting interaction with re cells and lysosomes: low doses of lipophiles appes to stabilize these and high doses to disrupt them The work of Burgen and Metcalfe with a model anaesthetic, benzyl alcohol, detectable by nuclear magnetic resonance, shows that the lipophile first interacts with and 'fluidifies' lipid of red cell mem brane, and later, with increasing concentrations, diss rupts the lipoprotein complex. We think we have encountered this in our high pressure experiments af Oxford. Mr Steve Daniels found that pulmonarg oedema occurred in mice decompressed from expoo sure to the fat-soluble compound $\mathbf{S F}_{6}$. Furthermoreo we have found that a range of anaesthetics, dissolved in saline and instilled intratracheally in guinea-pigs' excised lungs, produces 'solidification' of the lung, failure to recover instilled fluid (fig 5) and 5 release of a substance with the properties of a puE monary surfactant. This suggests that the anaesthetic breaks the lipoprotein link binding the lipid of surfactant in the alveoli.

Fourth, there is some evidence that anaesthe

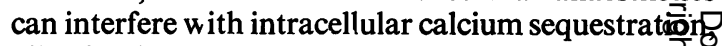
allowing its concentration to rise in the cytoplasm.

Finally, one must take account of the results of interaction with microsomes. Here, two response

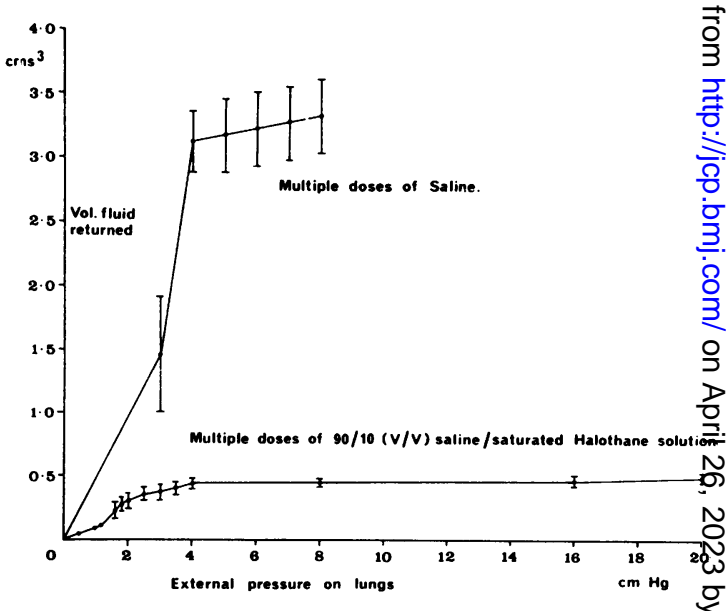

Fig 5 Effect of halothane on the recovery of saline instilled into the guinea pig isolated lungs. Abscissa, extrapleural pressure applied to lungs; ordinate, volume of fluid returned. Upper curve, saline control; lower curve after using saline $10 \%$ saturated with halothane. 
can occur, either inhibition, for instance by cannabidiol (Paton and Pertwee, 1972), or induction of activity, for instance by phenobarbitone. In either case a considerable range of secondary effects might be seen, for example, altered metabolism of endogenous steroid hormones, as well as alterations of the response to some external drug.

\section{Modulation of Specificity in the Action of a Lipophile}

So far, of course, it is implied that all lipophiles should be alike. It is obvious that they are not: even within the field of practical anaesthesia, the effects of ether and halothane are very different. Should a general approach be abandoned, or can other general factors be found to account for these differences? There are a number of these.

The first is the kinetics of a drug. A highly fatsoluble drug would not be expected to behave in quite the same way as one less so. Fat solubility goes hand in hand with water insolubility and protein binding, so that with a strong lipophile the concentration free in the plasma will be very low and tissue uptake will be both slow and blood flow limited. This alone could focus uptake on particular systems and hence bring out particular initial effects.

Second, metabolism sometimes changes the whole picture and converts a drug with seemingly nonspecific actions into one capable of specific chemical reaction; in particular, hydroxylation can give rise to the formation of highly reactive epoxides (these have been found with barbiturates and with tetrahydrocannabinol), which could react covalently with tissue constituents in still unknown ways. Another example is the observation that carbon tetrachloride can give rise to metabolites binding covalently with phospholipids (particularly phosphatidyl serine) in rat liver microsomes (Reynolds and Moslen, 1974). This seems to be a process with an important potential; a very small proportion of the drug so converted and bound (too small to be detected by clearance studies) could well accumulate to form a substantial amount of altered lipid.

It is interesting that the lipophilicity of the parent compound could direct where this takes place.

Third, it is too simple to suppose that the insertion of a lipophile into a hydrophobic site will be entirely independent of molecular shape and size. There was some justification for the idea since it has been found that the optical isomers of halothane are equiactive. But, with larger molecules it is already known from biophysical studies that their location in the membrane depends on molecular size and other features. Corresponding to this, for instance, the optical isomers of tetrahydrocannabinol are known to differ in potency in mice by a factor of 13 (Jones et al, 1974).

Lastly a rather special point should be mentioned. The forces between the molecules of a substance give it a self cohesiveness, and Hildebrand has shown how this may be characterized by what he termed $\delta$, the solubility parameter, a function of the energy of evaporation and the molar volume (see Hildeband $e t$ $a l, 1970$ ). When intermolecular forces are low (as in fluorinated hydrocarbons), evaporation is easy and $\delta$ is low. With strongly associated liquids, eg, ethanol, linked by hydrogen bonds, more energy is needed, and $\delta$ rises. The interesting point emerges that if $\delta$ differs too much between two compounds, they become immiscible; this is to say that if one is very much more self-cohesive than the other, it will prefer to associate with itself rather than with the molecules of the other compound and the latter will be extruded to form a separate phase. Therefore, there is another source of specificity, the physicochemical 'match' between the lipophilic solute and the lipid of membrane. In anaesthesia, for instance, there are reasons for believing that the properties of the membranes critical for acute effects and presumably synaptic, differ from those involved in chronic toxicity.

The argument may be summarized thus:

(a) A general distinction exists in pharmacology between 'specific' and 'nonspecific' action.

(b) This must be expected to apply in toxicology, and toxicity may not be related only to specific chemical groups with specific actions but also to more general physicochemical properties such as fat solubility with general actions.

(c) Taking anaesthetics and other lipophiles as a guide, one can tentatively suggest types of chronic toxicity that may occur. They include effects on cell division, on cell metabolism, on lipoprotein integrity, on calcium sequestration and on microsomal activity.

(d) Characteristically, chronic toxicity will be more important than acute toxicity.

(e) Although general patterns may be discernible, there are several mechanisms (kinetic, metabolic or physical) where differentiation between lipophiles will occur.

There are two final comments. The first is to reject false antitheses. It will have been noticed that even under 'nonspecific' action, specificity rears its head. It is also normal industrial practice to try to increase the potency of highly specific compounds by lipophilic loading, and extensive studies exist correlating potency of specific action with fat solubility. The issue is essentially operational and not of logical principle: sometimes it is better to consider specific factors and supplement them with nonspecific additions; sometimes it is more useful to begin with 
general physicochemical effects and introduce specific corrections. There is no need to waste time on semantics.

The second is the concept of a 'lipophilic' burden. It is important to ask whether toxicity of nonspecific type matters. I do not think we know. However, from experiments with anaesthetics and the amateur and industrial use of organic solvents, we can show that these substances interfere with cell turnover and with cell division. Moreover, if the huge mass of prescriptions of psychoactive drugs (some known to be unexpectedly prolonged in action) is considered, we may feel uneasy, particularly about effects on maturation of developing organisms. Since anaesthetics as a class are additive in their effects, we may need to think of a toxic 'lipophilic burden' made up of perhaps some petrol fumes, some household cleaning fluid, a little anaesthetic in hospital, a tranquillizer or two, a few sleeping tablets and some recreational lipophiles. It is, I think, largely speculation, but perhaps worth some attention, partly in trying to understand existing toxicity but also for instance, in the pharmaceutical field, where there may be a still unrecognized price to be paid for obtaining high potency by lipophilic loading.

\section{References}

Burgen, A. S. V., and Metcalfe, J. C. (1970). The application of nuclear magnetic resonance to pharmacological problems. J. Pharm Pharmacol., 22, 153-169.

Fink, B. R., Editor (1968). Toxicity of Anesthetics. Williams an Wilkins, Baltimore.

Halsey, M. J., Millar, R. A., and Sutton, J. A., Editors(1974). Molect lar Mechanisms in General Anaesthesia. Churchill Livingstorfe Edinburgh.

Hattula, M. L., and Karlog, O. (1973). Absorption and elimination of polychlorinated biphenyls (PCB) in goldfish. Acta pharmacef (kbh.), 32, 237-245.

Hildebrand, J. H., Prausnitz, J. M., and Scott, R. L. (1970). Regulä and Related Solutions. Van Nostrand-Reinhold, New York. 으

Jones, G., Pertwee, R. G., Gill, E. W., Paton, W. D. M., Nilsson, I M., Widman, M., and Agurell, S. (1974). Relative pharmacol8? gical potency in mice of optical isomers of $\triangle^{\prime}$-tetrahydrocap nabinol. Biochem. Pharmacol., 23, 439-446.

Lever, M. J., Miller, K. W., Paton, W. D. M., and Smith, E. B. (1970 Pressure reversal of anaesthesia. Nature (Lond.)., 231, 368-370

Mechoulam, R., Editor (1973). Marihuana; Chemistry, Pharms

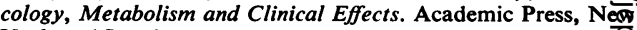
York and London.

Miller, K. W., Paton, W. D. M., Smith, E. B., and Smith, R. A. (1972) Physicochemical approaches to the mode of action of generg anesthetics. Anesthesiology, 36, 339-351.

Miller, K. W., Paton, W. D. M., Smith, R. A., and Smith, E. B. (197) The pressure reversal of general anesthesia and the crition volume hypothesis. Molec. Pharmacol., 9, 131-143.

Parke, D. V. (1968). The Biochemistry of Foreign Compounds. Pergamon, Oxford.

Paton, W. D. M., and Pertwee, R. G. (1972). Effect of cannabis and certain of its constituents on pentobarbitone sleeping time and phenazone metabolism. Brit. J. Pharmacol., 44, 250-261

Reynolds, E. S., and Moslen, M. T. (1974). In vivo covalent binding of ${ }^{14} \mathrm{CCI}_{4}$ metabolites in liver microsomal lipids. Biochen biophys. Res. Commun., 57, 747-750.

Singer, S. J., and Nicolson, G. L. (1972). The fluid mosaic model of the structure of cell membranes. Science, 175, 720-731.

Trudell, J. R., Hubbell, W. L., and Cohen, E. N. (1973). Pressure reversal of inhalation anesthetic-induced disorder in foin labeled phospholipid vesicles. Biochim. biophys. Acta (Amest.S, 291, 328-334. 